\title{
Erratum: Characterization of Gram matrices of multimode coherent states [Phys. Rev. A 99, 012346 (2019)]
}

\author{
Ashutosh S. Marwah and Norbert Lütkenhaus
}

(Received 22 February 2019; published 8 April 2019)

DOI: 10.1103/PhysRevA.99.049903

\section{INTRODUCTION}

In our paper, we derive criteria to decide whether a given Gram matrix can be realized using multimode coherent states. We use the entrywise complex logarithm function in those criteria. In our analysis, we did not consider the different branches of the complex logarithm function. In this Erratum, we correct this error. The results of our original article are recovered, although now a (finite) set of cases needs to be subjected to our criteria, rather than just a single one. One major resulting change is that, in our original paper, we provided a polynomial time (in the size of the matrix) algorithm to check if a matrix was a Gram matrix of coherent states or not, but now a similar algorithm would require exponential time. We have added a few results which can be used to efficiently decide this problem in certain cases.

\section{CORRECTIONS TO THE RESULTS IN THE SECTION: CHARACTERIZATION OF GRAM MATRICES OF MULTIMODE COHERENT STATES}

Suppose $P$ is a Gram matrix of multimode coherent states, i.e., $\left.\left.\left.\left.\left.\left.P=G\left(e^{i \phi_{1}} \| \alpha_{1}\right\rangle\right\rangle, e^{i \phi_{2}} \| \alpha_{2}\right\rangle\right\rangle, \ldots, e^{i \phi_{n}} \| \alpha_{n}\right\rangle\right\rangle\right)$ for some complex amplitude vectors $\left\{\alpha_{i}\right\}_{i=1}^{n} \subset \mathbb{C}^{m}$ and a set of real phases $\left\{\phi_{i}\right\}_{i=1}^{n} \subset \mathbb{R}$. Then, for every $i, j \in[n]$,

$$
\begin{aligned}
P_{i j} & \left.\left.\left.\left.=\left\langle e^{i \phi_{i}} \| \alpha_{i}\right\rangle\right\rangle, e^{i \phi_{j}} \| \alpha_{j}\right\rangle\right\rangle\right\rangle \\
P_{i j} & =\exp \left(-\frac{1}{2}\left(\left\|\alpha_{i}\right\|_{2}^{2}+\left\|\alpha_{j}\right\|_{2}^{2}-2\left\langle\alpha_{i}, \alpha_{j}\right\rangle\right)+i\left(\phi_{j}-\phi_{i}\right)\right),
\end{aligned}
$$

or equivalently,

$$
P_{i j}=\exp \left(-\frac{1}{2}\left\|\alpha_{i}-\alpha_{j}\right\|_{2}^{2}+i\left[\operatorname{Im}\left\{\left\langle\alpha_{i}, \alpha_{j}\right\rangle\right\}+\left(\phi_{j}-\phi_{i}\right)\right]\right) .
$$

However, this does not necessarily imply that

$$
\ln \left(P_{i j}\right)=-\frac{1}{2}\left(\left\|\alpha_{i}\right\|_{2}^{2}+\left\|\alpha_{j}\right\|_{2}^{2}-2\left\langle\alpha_{i}, \alpha_{j}\right\rangle\right)+i\left(\phi_{j}-\phi_{i}\right),
$$

or,

$$
\ln \left(P_{i j}\right)=-\frac{1}{2}\left\|\alpha_{i}-\alpha_{j}\right\|_{2}^{2}+i\left[\operatorname{Im}\left\{\left\langle\alpha_{i}, \alpha_{j}\right\rangle\right\}+\left(\phi_{j}-\phi_{i}\right)\right],
$$

since the complex ln function is not unique. Instead, for a particular branch of the $\ln$ function Eqs. (1) and (2) are equivalent to the existence of $\left\{N_{i j}\right\}_{i, j} \subset \mathbb{Z}$ for which

$$
\begin{aligned}
\ln \left(P_{i j}\right)= & -\frac{1}{2}\left(\left\|\alpha_{i}\right\|_{2}^{2}+\left\|\alpha_{j}\right\|_{2}^{2}-2\left\langle\alpha_{i}, \alpha_{j}\right\rangle\right) \\
& +i\left(\phi_{j}-\phi_{i}\right)+i 2 \pi N_{i j},
\end{aligned}
$$

or equivalently,

$$
\begin{aligned}
\ln \left(P_{i j}\right)= & -\frac{1}{2}\left\|\alpha_{i}-\alpha_{j}\right\|_{2}^{2}+i\left[\operatorname{Im}\left\{\left\langle\alpha_{i}, \alpha_{j}\right\rangle\right\}\right. \\
& \left.+\left(\phi_{j}-\phi_{i}\right)+2 \pi N_{i j}\right]
\end{aligned}
$$

holds for every $i, j \in[n]$.

A simple example where this error manifests itself is the following. Consider the Gram matrix $P:=G(|\alpha\rangle,-|\alpha\rangle)$, where $|\alpha\rangle$ is a coherent state, that is

$$
P=\left(\begin{array}{cc}
1 & -1 \\
-1 & 1
\end{array}\right) \text {. }
$$

Clearly $P \in G\left(\mathscr{C}_{2}^{2}\right)$, but for the ln function with the branch $[-\pi, \pi)$, we have

$$
\ln \odot P=\left(\begin{array}{cc}
0 & -i \pi \\
-i \pi & 0
\end{array}\right) .
$$

The matrix $\ln \odot P$ is not even Hermitian. If we were to use the characterization given in our original paper, then we would conclude erroneously that this matrix is not a Gram matrix of coherent states. In this Erratum, we will fix this error. We treat the original Theorem 1 of our paper as Lemma 1 here. We will use this as an intermediate step in the characterization of Gram matrices of coherent states. Before moving on to the lemma, recall the following definitions:

$$
\begin{aligned}
u & :=\left(\begin{array}{lll}
1 & \cdots 1
\end{array}\right) \\
{[\ln \odot(P)]_{i j} } & :=\ln P_{i j} .
\end{aligned}
$$

Lemma 1. For a matrix $Q \in \mathrm{L}\left(\mathbb{C}^{n}\right)$, the following are equivalent:

(I) There exists $m \in \mathbb{N}$, a set of complex vectors $\left\{\alpha_{i}: i \in[n]\right\} \subset \mathbb{C}^{m}$, and a set of real phases $\left\{\phi_{i}: i \in[n]\right\} \subseteq \mathbb{R}$ such that for $i, j \in[n]$,

$$
Q_{i j}=-\frac{1}{2}\left(\left\|\alpha_{i}\right\|_{2}^{2}+\left\|\alpha_{j}\right\|_{2}^{2}-2\left\langle\alpha_{i}, \alpha_{j}\right\rangle\right)+i\left(\phi_{j}-\phi_{i}\right) \text {. }
$$

(II) $Q \in \operatorname{Herm}\left(\mathbb{C}^{n}\right), Q_{i i}=0$ for all $i \in[n]$ and

$$
\left(\mathbb{1}-\frac{u u^{\dagger}}{n}\right) Q\left(\mathbb{1}-\frac{u u^{\dagger}}{n}\right) \geqslant 0 .
$$

Proof. This is simply a special (but completely equivalent) case of Theorem 1 of our original paper. The proof of this fact remains exactly the same as the proof of the statements (III) and (IV) in Theorem 1 of our original paper. All one needs to do is to replace $\ln \odot(P)$ with $Q$ in those proofs. In this lemma, we include the facts that the matrix $Q$ is Hermitian and that $Q_{i i}=0$ for every $i$ in the second statement itself since these 
are implied by the first one. Furthermore, all the rest of the statements of Theorem 1 of our paper also hold true if we replace statement $(\mathrm{I})$ of our original theorem with statement (I) of our present lemma, $\ln \odot(P)$ with $Q$ and add the conditions: $Q \in \operatorname{Herm}\left(\mathbb{C}^{n}\right)$ and $Q_{i i}=0$ for every $i$ in those statements as well. We have omitted these statements here for the sake of brevity.

Now, consider a complex ln function with the branch $[\beta, \beta+2 \pi)$ for some arbitrary but fixed choice of $\beta$. As stated earlier, a matrix $P$ satisfies $P \in G\left(\mathscr{C}_{m}^{n}\right)$ if and only if there exist some complex amplitude vectors $\left\{\alpha_{i}\right\}_{i=1}^{n} \subset \mathbb{C}^{m}$, a set of real phases $\left\{\phi_{i}\right\}_{i=1}^{n} \subseteq \mathbb{R}$, and a matrix of integers $N \in \mathbb{Z}^{n \times n}$ such that

$$
\begin{aligned}
& \ln \left(P_{i j}\right)-2 \pi i N_{i j} \\
& \quad=-\frac{1}{2}\left(\left\|\alpha_{i}\right\|_{2}^{2}+\left\|\alpha_{j}\right\|_{2}^{2}-2\left\langle\alpha_{i}, \alpha_{j}\right\rangle\right)+i\left(\phi_{j}-\phi_{i}\right) .
\end{aligned}
$$

Given a matrix $N$, we can use Lemma 1 to check if $Q:=\ln \odot(P)-2 \pi i N$ can be written in the form above. Therefore, we have the following equivalence for $P \in \operatorname{Herm}\left(\mathbb{C}^{n}\right)$ such that $P_{i i}=1$ for every $i$ : There exists $m \in \mathbb{N}$ such that $P \in G\left(\mathscr{C}_{m}^{n}\right)$ if and only if there exists a matrix of integers $N \in \mathbb{Z}^{n \times n}$ such that $[\ln \odot(P)-2 \pi i N] \in$ $\operatorname{Herm}\left(\mathbb{C}^{n}\right), 2 \pi i N_{i i}=\ln (1)$ for every $i$ and

$$
\left(\mathbb{1}-\frac{u u^{\dagger}}{n}\right)[\ln \odot(P)-2 \pi i N]\left(\mathbb{1}-\frac{u u^{\dagger}}{n}\right) \geqslant 0 .
$$

Note that this way we ensure that $[\ln \odot(P)-2 \pi i N]$ is Hermitian and hence we circumvent the problem we pointed out earlier in Eq. (5). However, in this form, this condition cannot be checked since there are countably infinite choices for the matrix $N$. Now, we will show that we only need to check this condition for a finite number of matrices $N$. First, we reiterate a fact as Lemma 2 which was mentioned in the original paper as well.

Lemma 2. If $\left.\left.\left.\left.\left.\left.P=G\left(e^{i \phi_{1}} \| \alpha_{1}\right\rangle\right\rangle, e^{i \phi_{2}} \| \alpha_{2}\right\rangle\right\rangle, \ldots, e^{i \phi_{n}} \| \alpha_{n}\right\rangle\right)\right)$, then $\left.\left.\left.\left.\left.\left.\quad P=G\left(e^{i \phi_{1}^{\prime}} \| 0\right\rangle\right\rangle, e^{i \phi_{2}^{\prime}} \| \alpha_{2}^{\prime}\right\rangle\right\rangle, \ldots, e^{i \phi_{n}^{\prime}} \| \alpha_{n}^{\prime}\right\rangle\right\rangle\right)$, where $\alpha_{i}^{\prime}=\alpha_{i}-\alpha_{1}$, and $\phi_{i}^{\prime}=\phi_{i}-\operatorname{Im}\left\{\left\langle\alpha_{i}, \alpha_{1}\right\rangle\right\}$ for every $i \in[n]$.

Proof. This can be proven by just plugging in the values given above in Eq. (2).

Our goal is to restrict the values the matrix element $N_{i j}$ can take. We accomplish this by using a complex ln function with the branch $[\beta, \beta+2 \pi)$. For this function, the imaginary part on the left-hand side of Eq. (4) is restricted to the interval $[\beta, \beta+2 \pi)$. If we can bound the terms in the imaginary part on the right-hand side, then we would be able to bound the terms $N_{i j}$ as well. For this purpose, we define the parameter,

$$
\delta:=\min _{i, j}\left|P_{i j}\right|
$$

If $\delta=0$, then we know that $P \notin G\left(\mathscr{C}_{m}^{n}\right)$ since the inner product between two coherent states cannot be zero [see Eq. (1)]. So, we consider $\delta \neq 0$. If $P \in G\left(\mathscr{C}_{m}^{n}\right)$, then $\left.\left.\left.\left.\left.\left.P=G\left(e^{i \phi_{1}} \| \alpha_{1}\right\rangle\right\rangle, e^{i \phi_{2}} \| \alpha_{2}\right\rangle\right\rangle, \ldots, e^{i \phi_{n}} \| \alpha_{n}\right\rangle\right\rangle\right)$ for some amplitude vectors $\left\{\alpha_{i}\right\}_{i=1}^{n}$ with $\alpha_{1}=0$ and real phases $\left\{\phi_{i}\right\}_{i=1}^{n}$ (using Lemma 2). Moreover, we can assume that for every $i \in$ $[n], \phi_{i} \in[0,2 \pi)$. Then, for every $i, j \in[n]$, we have that

$$
-2 \pi \leqslant \phi_{j}-\phi_{i} \leqslant 2 \pi \text {. }
$$

For a matrix $P \in G\left(\mathscr{C}_{m}^{n}\right)$, we have

$$
\begin{aligned}
\delta & =\min _{i, j}\left\{\exp \left(-\frac{1}{2}\left\|\alpha_{i}-\alpha_{j}\right\|_{2}^{2}\right)\right\} \\
& =\exp \left[-\frac{1}{2}\left(\max _{i, j}\left\{\left\|\alpha_{i}-\alpha_{j}\right\|_{2}\right\}\right)^{2}\right] .
\end{aligned}
$$

This implies that

$$
\max _{i, j}\left\{\left\|\alpha_{i}-\alpha_{j}\right\|_{2}\right\}=[-2 \ln (\delta)]^{1 / 2} .
$$

For every $i \in[n]$, and amplitude vector $\alpha_{i}$ in this representation of $P$, we have

$$
\begin{aligned}
\left\|\alpha_{i}\right\|_{2} & =\left\|\alpha_{i}-0\right\|_{2} \\
& =\left\|\alpha_{i}-\alpha_{1}\right\|_{2} \\
& \leqslant \max _{i, j}\left\{\left\|\alpha_{i}-\alpha_{j}\right\|_{2}\right\}=[-2 \ln (\delta)]^{1 / 2} .
\end{aligned}
$$

Furthermore, we have that for every $i, j \in[n]$,

$$
\begin{aligned}
\left|\left\langle\alpha_{i}, \alpha_{j}\right\rangle\right| & \leqslant\left\|\alpha_{i}\right\|_{2}\left\|\alpha_{j}\right\|_{2} \\
& \leqslant-2 \ln (\delta)=|2 \ln (\delta)|,
\end{aligned}
$$

since $\delta \in(0,1]$. We will use this to bound $\operatorname{Im}\left\{\alpha_{i}, \alpha_{j}\right\}$ using

$$
\left|\operatorname{Im}\left\{\left\langle\alpha_{i}, \alpha_{j}\right\rangle\right\}\right| \leqslant\left|\left\langle\alpha_{i}, \alpha_{j}\right\rangle\right| \leqslant|2 \ln (\delta)| .
$$

Now, observe that in Eq. (4) if we consider the ln function with the $[\beta, \beta+2 \pi)$ branch for every $i, j \in[n]$ we have

$$
\begin{aligned}
\beta & \leqslant 2 \pi N_{i j}+\operatorname{Im}\left\{\left\langle\alpha_{i}, \alpha_{j}\right\rangle\right\}+\left(\phi_{j}-\phi_{i}\right) \\
& \Rightarrow \beta-\operatorname{Im}\left\{\left\langle\alpha_{i}, \alpha_{j}\right\rangle\right\}-\left(\phi_{j}-\phi_{i}\right) \leqslant 2 \pi N_{i j} \\
& \Rightarrow \beta-|2 \ln (\delta)|-2 \pi \leqslant 2 \pi N_{i j},
\end{aligned}
$$

and,

$$
\begin{aligned}
2 \pi N_{i j} & +\operatorname{Im}\left\{\left\langle\alpha_{i}, \alpha_{j}\right\rangle\right\}+\left(\phi_{j}-\phi_{i}\right)<\beta+2 \pi \\
& \Rightarrow 2 \pi N_{i j}<\beta+2 \pi-\operatorname{Im}\left\{\left\langle\alpha_{i}, \alpha_{j}\right\rangle\right\}-\left(\phi_{j}-\phi_{i}\right) \\
& \Rightarrow 2 \pi N_{i j}<\beta+4 \pi+|2 \ln (\delta)|
\end{aligned}
$$

for which we have used Eqs. (10) and (11). Thus, for every $i, j \in[n]$, we have the following bound:

$$
N_{i j} \in \mathbb{Z} \cap\left[\frac{\beta}{2 \pi}-\frac{1}{\pi}|\ln (\delta)|-1, \frac{\beta}{2 \pi}+\frac{1}{\pi}|\ln (\delta)|+2\right) .
$$

To summarize, we have proven that if $P \in G\left(\mathscr{C}_{m}^{n}\right)$, then there exists an integer matrix $N \in \mathbb{Z}^{n \times n}$ with elements in the range given by Eq. (12) and a set of vectors $\left\{\alpha_{i}\right\}_{i}$ (where $\alpha_{1}=0$ specifically) and phases $\left\{\phi_{i}\right\}_{i}$ such that for every $i, j$,

$$
\begin{aligned}
& \ln \left(P_{i j}\right)-i 2 \pi N_{i j} \\
& \quad=-\frac{1}{2}\left(\left\|\alpha_{i}\right\|_{2}^{2}+\left\|\alpha_{j}\right\|_{2}^{2}-2\left\langle\alpha_{i}, \alpha_{j}\right\rangle\right)+i\left(\phi_{j}-\phi_{i}\right) .
\end{aligned}
$$

Conversely, if the above equation is satisfied for some integer matrix with the elements in the aforementioned range then $P \in G\left(\mathscr{C}_{m}^{n}\right)$. Using the characterization of matrix equations of this form given in Lemma 1 (of the present Erratum), we have shown that $P \in G\left(\mathscr{C}_{m}^{n}\right)$ if and only if there exists an integer matrix $N \in \mathbb{Z}^{n \times n}$ such that $[\ln \odot(P)-$ $2 \pi i N] \in \operatorname{Herm}\left(\mathbb{C}^{n}\right), 2 \pi i N_{i i}=\ln \left(P_{i i}\right)=\ln (1)$ for every 
$i \in[n]$, and

$$
\left(\mathbb{1}-\frac{u u^{\dagger}}{n}\right)[\ln \odot(P)-2 \pi i N]\left(\mathbb{1}-\frac{u u^{\dagger}}{n}\right) \geqslant 0 .
$$

Thus, we have proven the following result, which is a modified version of Theorem 1 of our original paper. Here, we consider the $\ln$ function with the branch $[\beta, \beta+2 \pi)$ for an arbitrary but fixed $\beta \in \mathbb{R}$.

Theorem 1. For a matrix $P \in \operatorname{Herm}\left(\mathbb{C}^{n}\right)$ such that $P_{i i}=1$ for all $i \in[n] . P \in G\left(\mathscr{C}_{m}^{n}\right)$ for some $m \in \mathbb{N}$ if and only if there exists an integer matrix $N \in \mathbb{Z}^{n \times n}$ such that $[\ln \odot(P)-2 \pi i N] \in \operatorname{Herm}\left(\mathbb{C}^{n}\right), 2 \pi i N_{i i}=\ln (1)$ for every $i \in$ $[n]$, and

$$
\left(\mathbb{1}-\frac{u u^{\dagger}}{n}\right)[\ln \odot(P)-2 \pi i N]\left(\mathbb{1}-\frac{u u^{\dagger}}{n}\right) \geqslant 0 .
$$

Furthermore, we can restrict the range of the elements of $N$ to the following:

$$
N_{i j} \in \mathbb{Z} \cap\left[\frac{\beta}{2 \pi}-\frac{1}{\pi}|\ln (\delta)|-1, \frac{\beta}{2 \pi}+\frac{1}{\pi}|\ln (\delta)|+2\right)
$$

for every $i, j \in[n]$, where $\delta:=\min _{i, j}\left|P_{i j}\right|>0$.

Note that for a given matrix $P$ we only need to check Eq. (13) for finitely many matrices $N$. However, we need to check the conditions for $\exp \left[O\left(n^{2}\right)\right]$ number of matrices $N$, where $n$ is the size of the matrix $P$. In our original paper, we claimed that using our method we can check if a matrix lies in $G\left(\mathscr{C}_{n}^{n}\right)$ in polynomial time. We would like to correct our claim. An algorithm based on a direct implementation of Theorem 1 will require exponential time. It might be possible to use semidefinite programming and rounding methods to create a more efficient algorithm for this task, but we do not pursue this lead here.

The rest of the claims and proofs in Sec. III of our original paper remain identical, even though one needs to consider the $2 \pi i N$ term in the equations.

Since, it seems computationally hard to decide if a Gram matrix $P$ lies in $G\left(\mathscr{C}_{n}^{n}\right)$ or not, we provide two corollaries, which would be helpful in certain cases. Both of these rely on the fact that it is easy to check if a matrix is a Euclidean distance matrix (EDM) [1,2]. Recall that a matrix $D \in L\left(\mathbb{C}^{n}\right)$ is a Euclidean distance matrix if there exist some set of vectors $\left\{v_{k}\right\}_{k=1}^{n} \subset \mathbb{R}^{n}$ such that

$$
D_{i j}:=-\frac{1}{2}\left\|v_{i}-v_{j}\right\|_{2}^{2}
$$

for every $i, j \in[n]$.

Corollary 1. If $P \in G\left(\mathscr{C}_{n}^{n}\right)$, then the matrix $R:=$ $\left[\ln \left(\left|P_{i j}\right|\right)\right]_{i j}$ is a Euclidean distance matrix (the ln function considered here is the real logarithm function).

Proof. If $P \in G\left(\mathscr{C}_{n}^{n}\right)$, then for every $i, j \in[n]$,

$$
\ln \left(\left|P_{i j}\right|\right)=-\frac{1}{2}\left\|\alpha_{i}-\alpha_{j}\right\|_{2}^{2}
$$

for some complex vectors $\left\{\alpha_{k}\right\}_{k=1}^{n} \subset \mathbb{C}^{n}$. Define, a $2 n$ dimensional vector $\beta_{i}:=\left(\operatorname{Re}\left\{\alpha_{i}\right\}, \operatorname{Im}\left\{\alpha_{i}\right\}\right)$ for every $i \in[n]$. These real vectors would also satisfy Eq. (15). Furthermore, these can be rotated into a $n$-dimensional space using an orthogonal matrix, which would leave the distance between these vectors invariant. Hence, the matrix $R$ would be an EDM.
One may wonder if the converse also holds in Corollary 1, i.e., given that for a Gram matrix $P$, the matrix $R:=\left[\ln \left(\left|P_{i j}\right|\right)\right]_{i j}$ is a Euclidean distance matrix, does this imply $P \in G\left(\mathscr{C}_{n}^{n}\right)$ ? This is seen not to be the case. A counterexample is the Gram matrix of three equiangular vectors in two dimensions,

$$
P=\left(\begin{array}{ccc}
1 & -0.5 & -0.5 \\
-0.5 & 1 & -0.5 \\
-0.5 & -0.5 & 1
\end{array}\right)
$$

The matrix $R:=\left[\ln \left(\left|P_{i j}\right|\right)\right]_{i j}$ in this case is an EDM, but one can use Theorem 1 to show that $P \notin G\left(\mathscr{C}_{n}^{n}\right)$. However, the converse does hold when all the elements of the Gram matrix $P$ are real and positive. This result is stated as Corollary 2 .

Corollary 2. If $P \in \operatorname{Herm}\left(\mathbb{C}^{n}\right)$ and $P_{i j} \in \mathbb{R}$ and $P_{i j}>0$ for every $i, j \in[n]$, then $P \in G\left(\mathscr{C}_{n}^{n}\right)$ if and only if $\ln \odot P$ is a Euclidean distance matrix (the ln function considered here is the real logarithm function).

Proof. The fact that, if $P \in G\left(\mathscr{C}_{n}^{n}\right)$, then $\ln \odot P$ is a Euclidean distance matrix can be seen using Corollary 1 (of the Erratum). For the other direction since $\ln \odot P$ is an EDM, we can choose vectors $\left\{\alpha_{i}\right\}_{i=1}^{n} \subset \mathbb{R}^{n}$ such that for every $i, j \in[n]$,

$$
\begin{aligned}
\ln P_{i j} & =-\frac{1}{2}\left\|\alpha_{i}-\alpha_{j}\right\|_{2}^{2} \\
& \Leftrightarrow P_{i j}=\exp \left(-\frac{1}{2}\left\|\alpha_{i}-\alpha_{j}\right\|_{2}^{2}\right),
\end{aligned}
$$

which implies that $\left.\left.\left.\left.P=G\left(\| \alpha_{1}\right\rangle\right\rangle, \ldots, \| \alpha_{n}\right\rangle\right\rangle\right)$ using the fact that $\alpha_{i}$ 's are real vectors and Eq. (2).

\section{CORRECTIONS TO PROOFS OF THE RESULTS IN THE SECTION: CLOSURE OF THE SET OF GRAM MATRICES OF MULTIMODE COHERENT STATES}

Lemma 1 and Theorem 2 of our original paper remain the same and are proved in exactly the same fashion. We only need to modify the proof of Lemma 2 of our original paper in this section. The statement of the lemma remains exactly the same, but we need to consider the integer matrices $N$ in the proof as well. The lemma is restated below as Lemma 3 .

Lemma 3. For $P \in \operatorname{Herm}\left(\mathbb{C}^{n}\right)$ such that $P_{i i}=1$ for all $i \in[n]$, if $P_{i j} \neq 0$ for all $i, j \in[n]$, then $P \in \overline{G\left(\mathscr{C}_{n}^{n}\right)}$ if and only if $P \in G\left(\mathscr{C}_{n}^{n}\right)$.

We adapt our original proof, which was based on using continuity, to prove this statement. The idea is that for a sequence, $\left\{P_{k}\right\}_{k=1}^{\infty} \subset G\left(\mathscr{C}_{n}^{n}\right)$ converging to $P$, we can restrict the possible integer matrices for each element of the sequence $P_{k}$ to a finite number of matrices for sufficiently large $k$. Since there are only a finite number of integer matrices for the entire sequence, one of the integer matrices must satisfy Eq. (13) for infinitely many elements of the sequence. We choose the subsequence of $\left\{P_{k}\right\}_{k=1}^{\infty}$ corresponding to this integer matrix and then apply continuity to prove the lemma. The reason the original proof does not work is that the integer matrices corresponding to the elements of the sequence may not converge themselves.

Proof of Lemma 3. Once again, the direction $P \in G\left(\mathscr{C}_{n}^{n}\right)$ implies $P \in \overline{G\left(\mathscr{C}_{n}^{n}\right)}$ is trivial. For the other direction, consider a sequence $\left\{P_{k}\right\}_{k=1}^{\infty} \subset G\left(\mathscr{C}_{n}^{n}\right)$ converging to $P$. First, observe that $P \in \operatorname{Pos}\left(\mathbb{C}^{n}\right)$ and that $P_{i i}=1$ for all $i \in[n]$ since these sets are closed. We choose the branch of the function $\ln$ such 
that it is continuous at $P_{i j}$ for every $i, j \in[n]$. Moreover, there exist integer matrices $\left\{N_{k}\right\}_{k=1}^{\infty} \subset \mathbb{Z}^{n \times n}$ for which each $\left[\ln \odot\left(P_{k}\right)-2 \pi i N_{k}\right] \in \operatorname{Herm}\left(\mathbb{C}^{n}\right), 2 \pi i\left(N_{k}\right)_{i i}=\ln (1) \quad$ for every $i$ and such that for all $y \in \mathbb{C}^{n}$ for which $\langle u, y\rangle=0$ we have (we are using an alternate characterization of Theorem 1 of our present Erratum; see original paper Theorem 1, Statement V),

$$
y^{\dagger}\left[\ln \odot\left(P_{k}\right)-2 \pi i N_{k}\right] y \geqslant 0 .
$$

Furthermore, we can assume that for all $i, j$,

$$
\left(N_{k}\right)_{i j} \in \mathbb{Z} \cap\left[\frac{\beta}{2 \pi}-\frac{1}{\pi}\left|\ln \left(\delta_{k}\right)\right|-1, \frac{\beta}{2 \pi}+\frac{1}{\pi}\left|\ln \left(\delta_{k}\right)\right|+2\right),
$$

where $\delta_{k}:=\min _{i, j}\left|\left(P_{k}\right)_{i j}\right|$. Before we proceed further, we show that for sufficiently large $k$ we can restrict the choice of matrices $N_{k}$ to a finite set.

Claim. For the sequence, $\left\{P_{k}\right\}_{k=1}^{\infty}$ and integer matrices $\left\{N_{k}\right\}_{k=1}^{\infty}$ as described above, there exists $M \in \mathbb{N}$ such that for all $k \geqslant M$ and for all $i, j \in[n]$, we have

$$
\left(N_{k}\right)_{i j} \in \mathbb{Z} \cap\left[\frac{\beta}{2 \pi}-\frac{1}{\pi}\left|\ln \left(\frac{\delta}{2}\right)\right|-1, \frac{\beta}{2 \pi}+\frac{1}{\pi}\left|\ln \left(\frac{\delta}{2}\right)\right|+2\right),
$$

where $\delta:=\min _{i, j}\left|P_{i j}\right|$. This essentially says that the range of elements of $N_{k}$ can be made independent of $k$.

Proof of claim. Since $\delta \neq 0$ and the elements $\left(P_{k}\right)_{i j} \rightarrow P_{i j}$ for every $i, j \in[n]$, we can choose $M$ such that for all $k \geqslant M$ and $i, j \in[n]$,

$$
\begin{aligned}
\|\left(P_{k}\right)_{i j}|-| P_{i j}|| & <\frac{\delta}{2} \\
& \Rightarrow\left|P_{i j}\right|-\frac{\delta}{2}<\left|\left(P_{k}\right)_{i j}\right| \\
& \Rightarrow \delta-\frac{\delta}{2}<\left|\left(P_{k}\right)_{i j}\right| \\
& \Rightarrow \frac{\delta}{2}<\left|\left(P_{k}\right)_{i j}\right| .
\end{aligned}
$$

Since this is true for all $i, j \in[n]$, for every $k \geqslant M$, we have

$$
\frac{\delta}{2}<\min _{i, j}\left|\left(P_{k}\right)_{i j}\right|=\delta_{k} \text {. }
$$

Using the fact that $|\ln (x)|$ is decreasing for $x \in(0,1]$, we see that

$$
\left[\frac{\beta}{2 \pi}-\frac{1}{\pi}\left|\ln \left(\delta_{k}\right)\right|-1, \frac{\beta}{2 \pi}+\frac{1}{\pi}\left|\ln \left(\delta_{k}\right)\right|+2\right)
$$

is a subset of

$$
\left[\frac{\beta}{2 \pi}-\frac{1}{\pi}\left|\ln \left(\frac{\delta}{2}\right)\right|-1, \frac{\beta}{2 \pi}+\frac{1}{\pi}\left|\ln \left(\frac{\delta}{2}\right)\right|+2\right) .
$$

This proves the claim for $M$ as chosen above.

Now, we only consider the infinite sequence of matrices $\left\{P_{k}: k \geqslant M\right\}$, where $M$ is the number as defined in the claim above. Observe that the integer matrix corresponding to each of these matrices $N_{k}$ is chosen from the finite set of matrices,

$$
\begin{aligned}
\mathcal{F} & :=\left\{N \in \mathbb{Z}^{n \times n} \mid \text { for all } i, j \in[n]: \frac{\beta}{2 \pi}-\frac{1}{\pi}\left|\ln \left(\frac{\delta}{2}\right)\right|-1\right. \\
& \left.\leqslant N_{i j}<\frac{\beta}{2 \pi}+\frac{1}{\pi}\left|\ln \left(\frac{\delta}{2}\right)\right|+2\right\} .
\end{aligned}
$$

We can write

$$
\mathcal{F}=\left\{K_{1}, K_{2}, \ldots, K_{l}\right\}
$$

for some $l \in \mathbb{N}$ to emphasize the fact that its finite. Since this set is finite and the sequence $\left(N_{k}\right)_{k}$ is infinite, there exists a $p \in[l]$ such that $N_{k}=K_{p}$ for infinitely many $k$. Define $K:=$ $K_{p}$ for convenience. We choose a subsequence of $\left\{P_{k}: k \geqslant\right.$ $M\}, \quad\left\{P_{k_{t}}\right\}_{t=1}^{\infty}$ such that $N_{k_{t}}=K$ for all $t \geqslant 1$. Also, as this is a subsequence of $\left\{P_{k}\right\}_{k}, P_{k_{t}} \rightarrow P$. Now, observe that for every $y \in \mathbb{C}^{n}$ such that $\langle u, y\rangle=0$ and for all $t \geqslant 1$,

$$
y^{\dagger}\left[\ln \odot\left(P_{k_{t}}\right)-2 \pi i K\right] y \geqslant 0 .
$$

This implies that for such a vector $y$,

$$
\begin{aligned}
& \lim _{t \rightarrow \infty} y^{\dagger}\left[\ln \odot\left(P_{k_{t}}\right)-2 \pi i K\right] y \geqslant 0 \\
& \Rightarrow y^{\dagger}\left\{\ln \odot\left[\lim _{t \rightarrow \infty}\left(P_{k_{t}}\right)\right]-2 \pi i K\right\} y \geqslant 0 \\
& \Rightarrow y^{\dagger}[\ln \odot(P)-2 \pi i K] y \geqslant 0,
\end{aligned}
$$

where we have used the fact that $K$ is a constant and the functions $-f_{y}(X)=y^{\dagger} X y$ and $f(X)=\ln \odot X$ are continuous. The $\ln \odot X$ function is continuous because we chose the branch of the $\ln$ function such that no element of $P$ lay on the branch cut. Furthermore, $2 \pi i K_{i i}=\ln (1)$ for every $i$ since $K=$ $N_{k}$ for some $k$, and $\ln \odot(P)-2 \pi i K=\lim _{t \rightarrow \infty}\left[\ln \odot\left(P_{k_{t}}\right)-\right.$ $2 \pi i K] \in \operatorname{Herm}\left(\mathbb{C}^{n}\right)$ because the set of Hermitian matrices is closed. Using an equivalent characterization of Theorem 1, this proves our claim.

\section{ACKNOWLEDGMENTS}

We would like to thank M. Kleinmann for independently pointing out the error in the original paper. We would also like to thank J. Watrous for a discussion regarding the possibility of reducing the time complexity of the algorithm to check if a matrix is a Gram matrix of coherent states. The work has been performed at the Institute for Quantum Computing, University of Waterloo, which is supported by Industry Canada. The research has been supported by NSERC under the Discovery Program, Grant No. 341495 and by the ARL CDQI Program.
[1] J. C. Gower, Euclidean distance geometry, Math. Sci. 7, 1 (1982).
[2] J. Dattorro, Convex Optimisation and Euclidean Distance Geometry (Meboo Publishing, Palo Alto, CA, 2017). 